\title{
Bacterial load of conditioned pressure ulcers is not a predictor for early flap failure in spinal cord injury
}

\author{
T Kamradt $^{1}$, S Klein ${ }^{2}$, S Zimmermann ${ }^{2}$, J Schröder-Braunstein ${ }^{3}$, CH Fürstenberg ${ }^{1,4}, \mathrm{C}_{\text {Hensel }}^{1}$, N Weidner ${ }^{1}$ \\ and $\mathrm{A} \mathrm{Hug}^{1}$
}

Objectives: Pressure ulcers impose a major lifetime medical problem to patients with high-grade spinal cord injury (SCl). For patients with stages 3-4 pressure ulcers, plastic surgery is often the only remaining treatment option. Despite considerable flap failure rates of around $30 \%$, only sparse knowledge exists on predictors for flap failure. Hence, identification of predictors for flap failures is needed. Methods: We prospectively enrolled $38 \mathrm{SCl}$ patients with stages 3-4 pressure ulcers scheduled for plastic surgery. Preoperative wound swabs, intraoperative tissue samples and postoperative drainage liquids were microbiologically analyzed. In multivariable logistic regression analyses, bacterial loads of deep tissue cultures of intraoperative samples as well as other clinical variables were analyzed with respect to the prediction of flap failures.

Results: The flap failure rate was $27.5 \%$. Bacterial loads of deep tissue cultures were not predictive for flap failure, neither was the colonization with a specific bacterial strain. We observed a considerable fluctuation of microbiological environment from initial swab cultures, intraoperative samples and postoperative drainage fluids. Antibioprophylaxis was sufficient in only $75 \%$ of deep tissue cultures and $69 \%$ of drainage fluids. Insufficient antibioprophylaxis was associated with a higher flap failure rates (odds ratio 6.3, confidence interval 1.2-41.0).

Conclusion: After inpatient wound conditioning, bacterial load analysis of intraoperative wound tissue cultures is ineffective in order to predict flap failure rates in SCI patients with stages 3-4 pressure ulcers after flap surgery. Instead, insufficient antibioprophylaxis might be a factor contributing to flap failure.

Spinal Cord (2017) 55, 535-539; doi:10.1038/sc.2016.186; published online 10 January 2017

\section{INTRODUCTION}

Pressure ulcers impose a major lifetime medical problem to patients with severe spinal cord injury (SCI). ${ }^{1,2}$ Despite prevention, ${ }^{3}$ lifetime prevalence as well as recurrence rates of pressure ulcers are still around $30 \%$ in the SCI population. ${ }^{1,4}$ Although SCI-associated mortality owing to wound sepsis and urosepsis decreased drastically after the introduction of antibiotics, ${ }^{5}$ the patient-level and socio-economic impact of pressure ulcers is still enormous (for example, quality of life, health-care costs). ${ }^{4,6,7}$

Although time consuming, conservative treatment is usually successful in the general aged population prone to pressure ulcers. ${ }^{8}$ In SCI, surgical treatment may be needed or prove beneficial with respect to general health, quality of life and the reduction of health-care costs in stage 3-4 ulcers. ${ }^{9}$ From a technical point of view, surgical repair comprises a variety of options such as primary wound closure and-in case of significant tissue loss-fasciocutaneous or musculocutaneous flaps. ${ }^{10}$

Proposed risk factors for flap failures and recurrent pressure ulcers in SCI are young age, low serum albumin, prior same-site surgery, smoking and poorly controlled diabetes. ${ }^{7,11}$ General health problems and inadequate patient behavior have been discussed as additional factors for recurrence. ${ }^{7,12,13}$ Despite great efforts in order to determine and reduce these risk factors as well as to improve surgical techniques, flap failure and recurrence rates of pressure ulcers remained almost unchanged. ${ }^{11,14-16}$

Inevitably, pressure ulcers are colonized by bacteria (that is, septic wounds). Even after surgical wound debridement, $90 \%$ of deep tissue cultures in the SCI population grow bacteria (Enterobacteriaceae $>$ Staphylococci $>$ Streptococci). ${ }^{17}$ Despite this knowledge, the level of evidence for a minimally required microbiological workup prior to surgical wound closure is moderate, and well-defined clinical guidelines for SCI patients do not exist. ${ }^{18}$ Consequently, quantitative microbiological testing prior to wound closure is uncommon in many SCI centers. In fact, the macroscopic wound inspection of the responsible surgeons and their belief of a proper wound situation for surgical closure decides the timing for plastic surgery. Our study is the first prospective analysis on the impact of quantitative deep tissue culture bacterial loads, obtained immediately before surgical wound closure, on early flap failure rates in SCI patients. To our knowledge, the present study represents the first prospective analysis on flap failure rates in SCI.

\section{MATERIALS AND METHODS}

Ethics statement

All study-related procedures were performed after obtaining informed consent according to protocols approved by the independent local ethics committee of the Medical Faculty of the University of Heidelberg.

\footnotetext{
${ }^{1}$ Spinal Cord Injury Center, Heidelberg University Hospital, Heidelberg, Germany; ${ }^{2}$ Department of Microbiology and Hygiene, Heidelberg University Hospital, Heidelberg, Germany; ${ }^{3}$ Department of Immunology, Heidelberg University Hospital, Heidelberg, Germany and ${ }^{4}$ Spinal Cord Injury Center, SRH Klinikum Karlsbad-Langensteinbach, Karlsbad, Germany Correspondence: Dr A Hug, Spinal Cord Injury Center, Heidelberg University Hospital, Schlierbacher Landstraße 200a, Heidelberg 69118, Germany.

E-mail: andreas.hug@med.uni-heidelberg.de

Received 19 January 2016; revised 29 October 2016; accepted 24 November 2016; published online 10 January 2017
} 


\section{Patients}

From January 2012 to May 2013, we prospectively enrolled 38 consecutive SCI patients with pressure ulcers stages 3-4 (according to the system proposed by the National Ulcer Advisory Panel; ${ }^{19}$ see Supplementary Table S1) admitted to our center for surgical repair.

In order to remove pressure ulcer load, all patients were immediately bed-immobilized, which led to the positive side effect of smoking cessation. Wound conditioning was started immediately after admission. For this purpose, all patients received at least one surgical wound debridement. Thereafter, wound dressings were performed at the discretion of the responsible wound manager (antiseptics: Polyhexanide gel, Prontosan (B. Braun Melsungen AG, Melsungen, Germany), Cutimed Sorbact (BSNmedical, Hamburg, Germany), patches: Allevyn Gentle Border, Allevyn Non-Adhesive (smith\&nephew, Hamburg, Germany)). Wound inspections were performed at least once daily by the responsible plastic surgeon. Decisions to schedule patients for musculocutaneous flap surgery were made at the discretion of the responsible plastic surgeon.

During flap surgery, the conditioned wound surface was stained with acid blue (Patentblau $5 \mathrm{mg} \mathrm{ml}^{-1}$ ) to visualize necrotic tissue. Then the blue colored superficial tissue was completely removed. After this final wound preparation, prophylactic intravenous antibiotic treatment was started. After 4-5 days, antibioprophylaxis was switched to an oral application for a total of 10 days. The standard antibiotic was cefuroxime (standard intravenous dose: $1500 \mathrm{mg}$ two times a day; standard oral dose: $500 \mathrm{mg}$ two times a day). In case preoperative swabs indicated cefuroxime resistance, other appropriate antibiotics were chosen (meropenem, imipenem/cilastatin, ciprofloxacin or amoxicillin/clavulanic acid). Depending on the pressure ulcer site, the following surgical flap techniques were applied: (1) sacral: gluteus maximus muscle flap; (2) ischial: biceps femoris muscle flap; and (3) trochanteric: tensor fasciae latae muscle flap. In selected cases, local musculocutaneous flaps with primary closure were performed at the discretion of the responsible plastic surgeon. At least one suction drain was placed, mobilized $2-3 \mathrm{~cm}$ after $48 \mathrm{~h}$ and removed $72 \mathrm{~h}$ after flap surgery. In case of excessive fluid drainage, drains were removed later, according to the surgeon's recommendation.

Immediately after flap surgery, all patients were transferred to dedicated alternating pressure antidecubitus mattresses (Profikair, Therakair, Kinetic Concepts Inc., Wiesbaden, Germany). Strict bed rest prohibiting any hip flexion was maintained for 21 days. Then surgical filaments were removed and hip flexion was gradually increased according to a standardized in-hospital schedule reaching $90^{\circ}$ of hip flexion on day 28 after flap surgery. In case of reddening or a small wound dehiscence, the schedule was prolonged at the discretion of the treating physician. Once $90^{\circ}$ of hip flexion was achieved, wheelchair mobilization was started and gradually extended according to an established in-hospital schedule. Close monitoring of the skin condition was performed during the wheelchair mobilization period.

A subgroup of the study population was part of a previously published study. ${ }^{20}$

\section{Blood/tissue samples}

C-reactive protein, serum albumin and hemoglobin Alc values were assessed on admission. After surgery, C-reactive protein, albumin and hemoglobin were assessed. As part of our in-hospital standard, wound swabs were routinely taken for microbiological testing toward the end of the wound-conditioning period. If surgical debridement was performed, swabs were taken after the final debridement prior to flap surgery. Routinely, the culture was taken with one swab, wiping across the whole diameter of the wound area.

During flap surgery, deep tissue biopsy specimens for the quantitative microbiological cultures were harvested under sterile conditions via the excision of a representative viable wound area (approximate weight of 8-10 g). The specimens were directly transferred into a dispersing device tube (DT20-M tube with rotor-stator element, VWR International GmbH, Darmstadt, Germany) filled with $10 \mathrm{ml} 0.9 \%$ sodium chloride. Tissue samples were homogenized using the IKA ULTRA-TURRAX Tube Drive control or manually (if insufficiently homogenized). Then $18 \mu \mathrm{l}$ of sample was plated using the Previ Isola (bioMérieux, Nürtingen, Germany) automated streaking machine. For each sample, Sheep Blood Agar (BD Diagnostics, Heidelberg, Germany),
Chocolate Agar (bioMérieux), MacConkey Agar (bioMérieux), Schädler Agar (bioMérieux), Kanamycin-Vancomycin Agar (bioMérieux) and Thioglycollate broth (BD Diagnostics) were inoculated. Blood, Chocolate, MacConkey Agar and Thioglycollate broth were incubated at $36^{\circ} \mathrm{C}$ and $5 \%$ carbondioxide. Schädler and Kanamycin-Vancomycin Agar were incubated under anaerobic conditions using BD Anaerobic Gaspak (BD Diagnostics). All cultures were incubated for at least $48 \mathrm{~h}$. If cultures remained sterile after $48 \mathrm{~h}$, they were incubated for 3 more days. Identification of germs was performed by smear preparation technique for MALDI-TOF MS (matrix-assisted laser desorption/ ionization-time of flight mass spectrometry; MicroFlex, Bruker, Bremen, Germany) or by biochemical identification using Vitek2 (bioMérieux) according to the manufacturer's instructions. Identification was accepted when scores were $>2.0$ for MALDI-TOF MS. For antimicrobial susceptibility testing, Vitek2 or Agar diffusion testing was used. EUCAST standards and breakpoints were applied.

For data analysis, bacterial colony-forming units (CFU) were recalculated per gram (g) wound tissue specimen. Postoperatively, wound drainage fluid was collected from suction drains $(10 \mathrm{ml}$, V-Monovette, Sarstedt, Nümbrecht, Germany) on the day of drainage removal. In case of bacterial growth, an antimicrobial susceptibility testing was performed as described above. In case of no bacterial growth, antibiotic treatment was regarded as being sufficient.

\section{Outcome measures and statistical analysis}

Categorical variables were summarized as counts and percentages. Medians with interquartile ranges (IQRs) and means with s.e.m. were used as a measure of central tendency for non-normally and normally distributed continuous variables, respectively. For statistical comparison of categorical variables, chi-square tests were used. For non-normally and normally distributed continuous variables, Wilcoxon rank sum test and $t$-tests were used, respectively.

For the primary outcome of the study, early flap failure as a binary variable was chosen. Early flap failure was defined as a local infection, necrosis or wound dehiscence with the need for a surgical re-intervention. The primary research hypothesis was that higher bacterial loads in tissue specimens during the flap surgery are predictive for early flap failure. For this analysis, bacterial load was entered as continuous predictor variable (CFU per g tissue) into a logistic regression model. Furthermore, we entered known predictors of flap failure (low serum albumin, poorly controlled diabetes, age, low postoperative hemoglobin level, prior same-site surgery ${ }^{7,11}$ ) as predictor variables into the regression model. Based on a 30\% flap failure rate, our study was statistically powered to detect a moderate-to-large standardized effect size. SAS 9.4 software was used for power calculations and statistical analyses (SAS, Cary, NC, USA).

\section{RESULTS}

\section{Demographic/clinical data}

In total, 38 patients were prospectively enrolled into the study with a total of 40 flap surgeries. Demographic and clinical data are summarized in Table 1. On admission, pressure ulcers lasted for a median of 5 (IQR 4-24) weeks. The mean wound-conditioning duration (primary debridement to wound closure) was 28 (IQR 18-42) days. In $12 / 40(30 \%)$ cases, prior same-site flap surgery was performed. Flap failures occurred in $11 / 40$ cases $(27.5 \%)$. In univariate analyses, no remarkable between-group differences (failure vs no failure) with respect to the neurological level of injury, known risk factors, ulcer stage, ulcer size, ulcer duration or the chosen flap technique were observed (Table 1).

\section{Technical aspects of flap surgery}

In $15 / 40(37.5 \%)$ cases, a standard musculocutaneous flap was regarded unnecessary by the responsible surgeon, and the wound was closed by a local musculocutaneous flap and primary closure. Eighteen gluteus maximus muscle flaps, four biceps femoris muscle flaps and three tensor fasciae latae muscle flaps were performed (Table 1). 
Table 1 Patient clinical data, ulcer characteristics and distribution of flap types

\begin{tabular}{|c|c|c|c|}
\hline \multirow{2}{*}{$\begin{array}{l}\text { Patient data }(\mathrm{n}=38, \\
\mathrm{n}=40 \text { flaps })\end{array}$} & Flap failure & Flap failure & \multirow[t]{2}{*}{ P-value } \\
\hline & No $(\mathrm{n}=29)$ & Yes $(\mathrm{n}=11)$ & \\
\hline Gender male, $n(\%)$ & $23(79.3)$ & $8(72.7)$ & 0.6861 \\
\hline Median age, years (IQR) & $58(38-66)$ & $52(42-67)$ & 0.9006 \\
\hline $\begin{array}{l}\text { Median ulcer duration, weeks } \\
\text { (IQR) }\end{array}$ & $5(4-32)$ & $5(5-10)$ & 0.8192 \\
\hline $\begin{array}{l}\text { Median time swab to tissue } \\
\text { culture, days (IQR) }\end{array}$ & $16(5-26)$ & $21(14-26)$ & 0.2334 \\
\hline $\begin{array}{l}\text { Median time flap surgery to } \\
\text { failure, days (IQR) }\end{array}$ & & $38(25-42)$ & \\
\hline $\begin{array}{l}\text { Mean intraoperative bacterial } \\
\text { load, } 10^{\mathrm{X}} \mathrm{CFU} \mathrm{g} \mathrm{g}^{-1} \pm \text { s.e.m. }\end{array}$ & $1.37(0.27)$ & $2.0(0.49)$ & 0.2672 \\
\hline Level of injury (para vs tetra) & & & 0.6632 \\
\hline Paraplegia, $n$ (row\%) & $23(79.3)$ & $8(20.7)$ & \\
\hline Tetraplegia, $n$ (row\%) & $6(66.7)$ & $3(27.3)$ & \\
\hline $\begin{array}{l}\text { AIS completeness (com vs } \\
\text { incom) }\end{array}$ & & & 0.3902 \\
\hline Complete lesion, $n$ (row\%) & $25(75.8)$ & $8(24.2)$ & \\
\hline Incomplete lesion, $n$ (row\%) & $4(57.1)$ & $3(42.9)$ & \\
\hline Ulcer classification (III vs IV) & & & 0.69 \\
\hline III, $n$ (row\%) & $9(81.8)$ & $2(18.2)$ & \\
\hline IV, $n$ (row\%) & $20(69.0)$ & $9(31.0)$ & \\
\hline Median ulcer size, $\mathrm{cm}^{2}$ (IQR) & $16(9-50)$ & $18(9-48)$ & 0.7716 \\
\hline $\begin{array}{l}\text { Median conditioning time, days } \\
\text { (IQR) }\end{array}$ & $28(18-42)$ & $28(18-36)$ & 0.7104 \\
\hline $\begin{array}{l}\text { Median bacterial load } \mathrm{CFU} \mathrm{g}^{-1} \\
\text { (IQR) }\end{array}$ & $10(0-100)$ & $10(0-10000)$ & 0.2606 \\
\hline $\mathrm{HbAlc} \%(I Q R)$ & $5.3(4.8-6.1)$ & $5.3(4.9-5.7)$ & 0.7718 \\
\hline Serum albumin, $g \mathrm{I}^{-1}(\mathrm{IQR})$ & $31.2(27.4-33.1)$ & $27.2(23.4-31.2)$ & 0.1263 \\
\hline C-reactive protein $\mathrm{mg} \mathrm{I}^{-1}(\mathrm{IQR})$ & $37.6(17.9-86.6)$ & $48.1(22.6-75.0)$ & 0.9396 \\
\hline Prior same-site surgery, $n(\%)$ & $9(31.0)$ & $5(45.5)$ & 0.3963 \\
\hline Type of flap (overall difference) & & & 0.3425 \\
\hline Primary closure, $n$ (column\%) & $12(41.4)$ & $3(27.3)$ & \\
\hline Gluteal muscle, $n$ (column\%) & $12(41.4)$ & $6(54.5)$ & \\
\hline Biceps muscle, $n$ (column\%) & $1(3.4)$ & $2(18.2)$ & \\
\hline
\end{tabular}

Abbreviations: AIS, American Spinal Injury Association (ASIA) Impairment Scale; CFU, colonyforming units; HbAlc, hemoglobin $\mathrm{Alc}$; IQR, interquartile range.

\section{Microbiological samples}

The spectrum of isolated bacteria from swab, intraoperative tissue specimen and postoperative drainage fluid cultures is shown in Tables 2 and 3. Preoperative swab cultures (available in $n=35$ wounds) revealed colonization with Enterobacteriacea (25/35, 71\%), Staphylococci $(22 / 35,63 \%)$, Streptococci and Enterococci (20/35, $57 \%)$. Anaerobic bacteria were found in 11/35 (31\%), and Pseudomonas aeruginosa was isolated in 6/35 (17\%). 18/35 (51\%) cultures were positive for three or more different bacterial species (Table 3). Intraoperative tissue specimens $(n=40)$ tested positive for the following bacteria: (1) Staphylococci $17 / 40$ (43\%), (2) Enterobacteriacea 13/40 (33\%), (3) Streptococci and Enterococci 8/40 (20\%), and (4) Pseudomonas aeruginosa 7/40 (18\%). Only 3/40 (7.5\%) cultures tested positive for three or more different species. Overall, bacterial loads were low after wound conditioning (median $10 \mathrm{CFU} \mathrm{g}^{-1}$, IQR 0-100). Bacterial loads were $10^{5} \mathrm{CFU} \mathrm{g}^{-1}$ in $2 / 40$ (5\%), $10^{4} \mathrm{CFU} \mathrm{g}^{-1}$ in $3 / 40$ (7.5\%), $10^{3} \mathrm{CFU} \mathrm{g}^{-1}$ in $4 / 40(10 \%)$, $10^{2} \mathrm{CFU} \mathrm{g}^{-1}$ in $7 / 40(17.5 \%)$ and $10^{1} \mathrm{CFU} \mathrm{g}^{-1}$ in $12 / 40$ (30\%). Also, $12 / 40(30 \%)$ specimen cultures showed no significant growth or were sterile. In one of the two cases with bacterial counts of $10^{5} \mathrm{CF} \mathrm{g}^{-1}$, sanious wound infection and consecutive flap failure occurred. Methicillin-resistant Staphylococcus aureus (MRSA) was found in intraoperative and drainage fluid samples. As it was not present before or not detected by the preoperative swab/tissue culture, the antibioprophylaxis with ciprofloxacin was insufficient. In the other $10^{5} \mathrm{CFU} \mathrm{g}^{-1}$ case (Pseudomonas aeruginosa), antibioprophylaxis with imipenem was also insufficient. However, wound healing was not affected negatively.

Drainage fluids were available from 30 patients. Bacterial growth was found in $15 / 30(50 \%)$. Of those, microbiological workup revealed Pseudomonas aeruginosa in 6/15 (40\%), Streptococci and Enterococci in 6/15 (40\%) and MRSA in 1/15 (6.7\%). For 9/15 (60\%) positive cultures, the identified bacteria were not susceptible to the administered antibioprophylaxis.

\section{Blood samples}

Median hemoglobin Alc levels on admission were 5.4\% (IQR 4.8-5.9). Only four patients showed elevated hemoglobin Alc levels, ruling out diabetes as a relevant risk factor in our study cohort. Median serum albumin levels on admission were $29 \mathrm{gl}^{-1}$ (IQR 25-32). 18/33 (55.5\%) of patients had levels below the lower limit of normal, and 8/33 (24.2\%) exhibited significant hypalbuminemia with values $<25 \mathrm{~g} \mathrm{l}^{-1}$. Median C-reactive protein levels on admission were $48.1 \mathrm{mg} \mathrm{l}^{-1}$ (IQR 21.1-78.5).

\section{Flap failure analysis}

With respect to the primary outcome measure, logistic regression analysis revealed that the intraoperative bacterial load was not predictive for flap failure (odds ratio 1.4, confidence interval 0.9-2.2 per CFU log-unit, $P=0.1867)$. Moreover, we could not identify certain bacterial species associated with early flap failure.

In secondary exploratory analyses, we identified an ineffective antibioprophylaxis for the identified intraoperative bacteria as a putative predictor for flap failure. In $5 / 10(50.0 \%)$ cases of the flap failure group, the intraoperative specimens revealed resistant bacteria for the administered antibiotics, as compared with $3 / 22(13.6 \%)$ in the non-failure group (odds ratio 6.3, confidence interval 1.2-41.0, $P=0.0320)$.

\section{DISCUSSION}

In SCI, high-stage pressure ulcers occur frequently with a cumulative lifetime risk of up to $50 \% .^{1,2,5}$ Patients with pressure ulcers scheduled for flap surgery often require time-consuming preoperative wound conditioning and postoperative skin conditioning (several weeks). Despite this meticulous perioperative management, early flap failures occur in up to $36 \%$ of cases, ${ }^{11,13}$ resulting in even longer length of hospital stays. Hence, early predictors for flap failure-before surgical closure of the wounds-are urgently needed.

In this prospective study on 38 SCI patients with high-stage pressure ulcers, we aimed to identify microbiological predictors for early flap failure. Applying in-hospital standardized perioperative care, however, (1) quantitative microbiological analysis of wound tissue specimens and (2) qualitative bacterial spectrum analysis were ineffective with respect to the prediction of early flap failure. 
Table 2 Identification of bacterial species in preoperative swabs, intraoperative tissue samples and postoperative drainage fluid

\begin{tabular}{lccc}
\hline Species & Swabs & Intraoperative & Drainage \\
& $(\mathrm{n}=35)$ & sample $(\mathrm{n}=40)$ & fluid $(\mathrm{n}=30)$
\end{tabular}

Enterobacteria

E. coli

Proteus mirabilis

Enterobacter cloacae

Enterobacter aerogenes

Klebsiella pneumoniae

Morganella morganii

Proteus vulgaris

Citrobacter koseri

Other

Other Gram-negative rods

Pseudomonas aeruginosa

Acinetobacter baumanii

Haemophilus parainfluenzae

Achromobacter denitrificans

Staphylococci

MRSA

MSSA

CNS

Streptococci and Enterococci

Enterococcus faecalis

Enterococcus faecium

Other Enterococci

Streptococcus $B$

Streptococci viridans

Anaerobics

Corynebacterium species

Bacillus species

Yeast

Candida sp.

Candida albicans
Other Streptococci (non-A, non-B)

\section{3}

3

1

2

2

3

1

1

6

4

1

1

6

$\begin{array}{rrr}1 & 1 & 1 \\ 12 & 14 & 3 \\ 9 & 2 & 1\end{array}$

Abbreviations: CNS, coagulase-negative Staphylococci; MRSA, methicillin-resistant

Staphylococcus aureus; MSSA, methicillin-susceptible Staphylococcus aureus.

The observed early flap failure rate of $27.5 \%$ in our study, which to our knowledge is the first prospective study in the literature, is compatible with published rates of 7-62\%. ${ }^{12,13,21,22}$ This wide range of failure rates in the literature may be partly attributed to different definition criteria for flap failures, for example, early vs late failure or recurrent ulcers. ${ }^{6,11}$ Our main criterion for flap failure was the need for a surgical re-intervention, even in case of a small necrotic area or wound dehiscence. A sensitivity analysis of the flap failure subgroup with putrid flap infection and a systemic inflammatory response $(n=3)$ did also not indicate a correlation between the intraoperative bacterial load and early flap failure.

Secondary exploratory analyses suggested that insufficient antibioprophylaxis might have a crucial role for an increased flap failure risk. Antibioprophylaxis-resistant bacterial species in intraoperative tissue specimens were associated with an increased flap failure rate. According to our in-hospital protocol, antibioprophylaxis was based on antibiograms from preoperative swab cultures (that is, bacteria of swab
Table 3 Microbiological culture results over the course of wound conditioning and flap surgery

\begin{tabular}{|c|c|c|c|c|c|c|}
\hline & \multirow[t]{2}{*}{$\begin{array}{l}\text { Culture } \\
\text { negative }\end{array}$} & \multicolumn{5}{|c|}{$\begin{array}{c}\text { Culture positive (different } \\
\text { bacterial species, (n)) }\end{array}$} \\
\hline & & 1 & 2 & 3 & 4 & $\geqslant 5$ \\
\hline Preoperative swabs $(n=35)$ & 2 & 3 & 12 & 12 & 5 & 1 \\
\hline Intraoperative samples $(n=40)$ & 7 & 19 & 11 & 3 & 0 & 0 \\
\hline Drainage fluid $(n=30)$ & 15 & 11 & 4 & 0 & 0 & 0 \\
\hline
\end{tabular}

cultures were sensitive to the applied antibioprophylaxis). With respect to the subsequent intraoperative tissue culture results, however, administered antibiotics were regarded insufficient in 8/32 (25.0\%) cases. Hence, significant bacterial spectrum fluctuations seem to occur between initial swab and intraoperative tissue cultures. The clinical problem of quantitative and qualitative bacterial spectrum fluctuations was already addressed before. ${ }^{17,23}$ Our data in SCI patients corroborate these findings and argue for the acquisition of deep tissue samples and bacterial susceptibility testing once wound conditioning is completed. The growth of resistant bacteria in 9/29 (31\%) drainage fluid cases underscores the importance of reassessing antibioprophylaxis during wound closure.

Of note, Pseudomonas aeruginosa was hardly affected (swabs 6/35, intraoperative $7 / 40$, drainage fluid $6 / 30$ ) by wound conditioning and antibioprophylaxis. Pseudomonas has only recently been linked to promote wound healing via secretion of tumor necrosis factor- $\alpha$ in an animal wound model. ${ }^{24}$ In our study, Pseudomonas was found in wounds of only $1 / 11$ (9\%) flap failures, but in 6/29 (21\%) specimens in the uncomplicated group, also suggesting a wound-healing effect. Additionally, in two of our patients with highest bacterial loads $\left(10^{5} \mathrm{CFU} \mathrm{g}^{-1}\right)$ and antibiotic-resistant bacteria, one with MRSA had a flap failure and one with Pseudomonas aeruginosa exhibited uncomplicated wound healing. On the other hand, there is clinical evidence for biofilm-producing Pseudomonas aeruginosa to inhibit wound healing in chronic wounds of non-SCI patients. ${ }^{25}$ At this point, the observation that specific bacterial wound colonization might be beneficial remains anecdotal. Future investigations may help to elucidate this observation in the SCI population.

Keys et al. ${ }^{11}$ retrospectively analyzed 138 pressure ulcer patients. One finding was a surgical revision rate of $36 \%$ owing to 'operative failure' (that is, suture dehiscence, infection or hematoma) in line with our definition criteria for early flap failure. In their analysis, younger age ( $<45$ years), prior same-site surgery and poor diabetes control were independent predictors of flap failure, which we could not reproduce in our prospective analysis.

Overall, we observed sufficient reductions of bacterial load/diversity in wound grounds after conditioning (median duration 28 days) applying our in-hospital standard protocol. After conditioning, wound bacterial loads were negligible in most cases. For this reason, bacterial load might have been ineffective for the prediction of early flap failure. However, bacterial load analysis might prove meaningful in order to shorten preoperative conditioning procedures. Our study was not designed to address this specific question. With respect to the enormous burden for patients as well as health-care providers, such a screening strategy definitely deserves further investigation.

Despite some authors arguing against primary closure, ${ }^{6,10}$ it was our operation of choice as a tissue-saving method in 15/40 (37.5\%) of our patients, and we did not observe an increased flap failure rate ( $P$-value: 0.4099). Our postoperative regime of avoiding tension on the 
suture for 3 weeks and gradually stretching the tissue for another week is quite long compared with standard procedures in other clinics but may give the tissue ample opportunity to elongate without any cutaneous or subcutaneous dehiscence.

Our study has several limitations. First, it was not statistically powered to detect small effect sizes. Hence, small effects could well have been missed. Still, our study is so far the largest prospective analysis regarding the impact of quantitative/qualitative microbiological tissue specimen analysis with respect to the prediction of early flap failures in the SCI population with high-stage pressure ulcers. The results of our study are representative for SCI centers treating SCI patients before and after flap surgery as inpatients. General transferability to other centers handling those patients as outpatients or in nursing facilities is limited. Moreover, as we performed a pragmatic study with no standardization of the actual surgical and non-surgical wound treatment procedures, generalizability to other treatment centers may be limited. Furthermore, our patients were treated with a limited set of flap techniques. Our findings are not easily transferrable to centers favoring other flap surgery techniques (for example, free flaps). Owing to the study design, we can only present data on early flap failures. We cannot comment on the impact of microbiological analysis on long-term flap survival rates.

In conclusion, after inpatient wound conditioning, bacterial load analysis of intraoperative wound tissue cultures is ineffective in predicting early flap failure rates in SCI patients with stage 3-4 pressure ulcers after flap surgery. However, insufficient antibioprophylaxis might be a contributing factor for flap failure. Further research is urgently needed to identify preventable or modifiable risk factors for early flap failures.

\section{DATA ARCHIVING}

There were no data to deposit.

\section{CONFLICT OF INTEREST}

The authors declare no conflict of interest.

\section{ACKNOWLEDGEMENTS}

This study was supported by a Grant of the Medical Faculty Heidelberg to Andreas Hug.

1 Chen Y, Devivo MJ, Jackson AB. Pressure ulcer prevalence in people with spinal cord injury: age-period-duration effects. Arch Phys Med Rehabil 2005; 86: 1208-1213.
2 Johnson RL, Gerhart KA, McCray J, Menconi JC, Whiteneck GG. Secondary conditions following spinal cord injury in a population-based sample. Spinal Cord 1998; 36 45-50.

3 Regan MA, Teasell RW, Wolfe DL, Keast D, Mortenson WB, Aubut JA et al. A systematic review of therapeutic interventions for pressure ulcers after spinal cord injury. Arch Phys Med Rehabil 2009; 90: 213-231.

4 Niazi ZB, Salzberg CA, Byrne DW, Viehbeck M. Recurrence of initial pressure ulcer in persons with spinal cord injuries. Adv Wound Care 1997; 10: 38-42.

5 Netscher D, Clamon J, Fincher L, Thompson R. Surgical repair of pressure ulcers. Plast Surg Nurs 1996; 16: 225-233 239.

6 Sorensen JL, Jorgensen B, Gottrup F. Surgical treatment of pressure ulcers. Am J Surg 2004; 188: 42-51.

7 Byrne DW, Salzberg CA. Major risk factors for pressure ulcers in the spinal cord disabled: a literature review. Spinal Cord 1996; 34: 255-263.

8 Brandeis GH, Morris JN, Nash DJ, Lipsitz LA. The epidemiology and natura history of pressure ulcers in elderly nursing home residents. JAMA 1990; 264 2905-2909.

9 Singh R, Singh R, Rohilla RK, Siwach R, Verma V, Kaur K. Surgery for pressure ulcers improves general health and quality of life in patients with spinal cord injury. J Spinal Cord Med 2010; 33: 396-400.

10 Bauer J, Phillips LG. MOC-PSSM CME article: Pressure sores. Plast Reconstr Surg 2008; 121(Suppl 1): 1-10.

11 Keys KA, Daniali LN, Warner KJ, Mathes DW. Multivariate predictors of failure after flap coverage of pressure ulcers. Plast Reconstr Surg 2010; 125: 1725-1734.

12 Mandrekas AD, Mastorakos DP. The management of decubitus ulcers by musculocutaneous flaps: a five-year experience. Ann Plast Surg 1992; 28: 167-174.

13 Disa JJ, Carlton JM, Goldberg NH. Efficacy of operative cure in pressure sore patients. Plast Reconstr Surg 1992; 89: 272-278.

14 Relander M, Palmer B. Recurrence of surgically treated pressure sores. Scand J Plast Reconstr Surg Hand Surg 1988; 22: 89-92.

15 Berry RB. The late results of surgical treatment of pressure sores in paraplegics. $\mathrm{Br} \mathrm{J}$ Surg 1980; 67: 473-474.

16 Goodman CM, Cohen V, Armenta A, Thornby J, Netscher DT. Evaluation of results and treatment variables for pressure ulcers in 48 veteran spinal cord-injured patients. Ann Plast Surg 1999; 42: 665-672.

17 Heym B, Rimareix F, Lortat-Jacob A, Nicolas-Chanoine MH. Bacteriological investigation of infected pressure ulcers in spinal cord-injured patients and impact on antibiotic therapy. Spinal Cord 2004; 42: 230-234.

18 Panel EPUAPaNPUA. Treatment of Pressure U/cers: Quick Reference Guide. National Pressure Ulcer Advisory Panel: Washington, DC, USA, 2009.

19 Black J, Baharestani M, Cuddigan J, Dorner B, Edsberg L, Langemo D et al. National Pressure Ulcer Advisory Panel's updated pressure ulcer staging system. Urol Nurs 2007; 27: 144-150 156

20 Kamradt T, Rasch C, Schuld C, Bottinger M, Murle B, Hensel C et al. Spinal cord injury: association with axonal peripheral neuropathy in severely paralysed limbs. Eur $\mathrm{J}$ Neurol 2013; 20: 843-848

21 Kirkby B, Holck S. [Surgical treatment of pressure sores using the gluteus maximus island flap. A new method]. Ugeskr Laeger 1982; 144: 2411-2414.

22 Tavakoli K, Rutkowski S, Cope C, Hassall M, Barnett R, Richards M et al. Recurrence rates of ischial sores in para- and tetraplegics treated with hamstring flaps: an 8-year study. Br J Plast Surg 1999; 52: 476-479.

23 Thornhill-Joynes M, Gonzales F, Stewart CA, Kanel GC, Lee GC, Capen DA et al. Osteomyelitis associated with pressure ulcers. Arch Phys Med Rehabil 1986; 67: 314-318.

24 Kanno E, Kawakami K, Ritsu M, Ishii K, Tanno H, Toriyabe S et al. Wound healing in skin promoted by inoculation with Pseudomonas aeruginosa PAO1: the critical role of tumor necrosis factor-alpha secreted from infiltrating neutrophils. Wound Repair Regen 2011; 19: 608-621.

25 James GA, Swogger E, Wolcott R, Pulcini E, Secor P, Sestrich J et al. Biofilms in chronic wounds. Wound Repair Regen 2008; 16: 37-44.

Supplementary Information accompanies this paper on the Spinal Cord website (http://www.nature.com/sc) 